\title{
Independent effects of adaptation and attention on perceived speed
}

\author{
Katharina Anton-Erxleben ${ }^{1,2}$, Katrin Herrmann ${ }^{1}$, and Marisa Carrasco ${ }^{1,2}$ \\ ${ }^{1}$ Psychology, New York University \\ ${ }^{2}$ Neural Science, New York University
}

\section{Abstract}

Adaptation and attention are two mechanisms by which sensory systems manage limited bioenergetic resources: Whereas adaptation decreases sensitivity for stimuli just previously encountered, attention increases sensitivity for behaviorally relevant stimuli. In the visual system, these changes in sensitivity are accompanied by a change in stimulus appearance of different dimensions, such as speed. Adaptation causes an underestimation, whereas attention leads to an overestimation of speed. Here we investigate whether the effects of these mechanisms interact and how they affect appearance. We tested the effects of adaptation and subsequent allocation of attention on perceived speed. A quickly moving adaptor decreased the perceived speed of subsequent stimuli, whereas a slow adaptor did not alter perceived speed. Attention increased perceived speed regardless of the adaptation effect, indicating that adaptation and attention affect perceived speed independently. Moreover, the finding that attention can alter perceived speed after adaptation indicates that adaptation is not merely neuronal fatigue.

\section{Introduction}

At every moment, we are exposed to an overwhelming amount of information. Due to limited neural resources, we cannot process all input equally well (Lennie, 2003). Several mechanisms, such as adaptation and attention, reduce the cost of cortical computation by shifting priority to relevant information.

Adaptation decreases sensitivity for stimuli that have been encountered repeatedly in the past, thereby increasing sensitivity to changes in the environment (Kohn, 2007). Adaptation is ubiquitous in sensory modalities including vision: we adapt to very diverse low- and highlevel stimulus features such as color, spatial frequency, motion, and contrast as well as aspect ratio and facial expression (Webster, 2011). Although adaptation had long been viewed as a byproduct of neuronal fatigue, where for a given neuron the level of fatigue depends on the magnitude of its response to the adapting stimulus, it is now clear that adaptation may have functional benefits (Kohn, 2007; Webster, 2011). Adaptation adjusts the dynamic range of the system by shifting contrast-response functions to match contrast levels prevalent in the environment (Movshon \& Lennie, 1979; Ohzawa, Sclar, \& Freeman, 1982). Adaptation also shifts tuning curves for orientation (Jin, Dragoi, Sur, \& Seung, 2005) and direction (Krekelberg, van Wezel, \& Albright, 2006) to optimize processing of prevailing stimulus features. Thus, adaptation optimizes processing resources for recent stimulus conditions. A consequence of the selective change in sensitivity is an aftereffect-a change in the appearance of visual stimulus features, e.g. spatial frequency (Blakemore, Nachmias, \& Sutton, 1970) and motion direction (Levinson \& Sekuler, 1976). Adaptation 
typically causes a repulsive shift in perception of a given feature away from the adaptor, either an over- or underestimation.

Attention is another mechanism by which sensory systems prioritize relevant information. Spatial attention enables us to dynamically concentrate processing resources at the location of interest, either overtly (by moving our gaze) or covertly (in the absence of eye movements). Spatial covert attention improves contrast sensitivity, spatial resolution and processing speed for stimuli at the attended location while it impairs performance at the unattended locations (Carrasco, Giordano, \& McElree, 2006; Carrasco, Penpeci-Talgar, \& Eckstein, 2000; Giordano, McElree, \& Carrasco, 2009; Herrmann, Montaser-Kouhsari, Carrasco, \& Heeger, 2010; Ling \& Carrasco, 2006; Lu \& Dosher, 1998; Luck et al., 1994; Montagna, Pestilli, \& Carrasco, 2009; Pestilli, Viera, \& Carrasco, 2007; Yeshurun \& Carrasco, 1998). Spatial covert attention also changes the appearance of visual stimulus features (Abrams, Barbot, \& Carrasco, 2010; Anton-Erxleben, Abrams, \& Carrasco, 2010; Anton-Erxleben, Henrich, \& Treue, 2007; Carrasco, Ling, \& Read, 2004; Fuller \& Carrasco, 2006; Gobell \& Carrasco, 2005; T. Liu, Abrams, \& Carrasco, 2009; Liu, Fuller, \& Carrasco, 2006; Montagna \& Carrasco, 2006; Störmer, McDonald, \& Hillyard, 2009), for example it leads to an overestimation of speed (Fuller, Park, \& Carrasco, 2009; Turatto, Vescovi, \& Valsecchi, 2007).

\section{How do adaptation and attention interact?}

It is established that attention during the adapting phase potentiates the effects of adaptation on a variety of visual phenomena; e.g., motion, orientation, and contrast aftereffects (Alais \& Blake, 1999; Lankheet \& Verstraten, 1995; Ling \& Carrasco, 2006; Liu, Larsson, \& Carrasco, 2007; but see Hudson, Schiff, Victor, \& Purpura, 2009), but little is known about whether and how adaptation modulates the effects of attention. The magnitude of the attention effect on contrast sensitivity does not vary with adaptation state (Pestilli et al., 2007). However, in a texture segmentation task adaptation to high- but not low- spatial frequencies diminished the effects of attention on performance (Carrasco, Loula, \& Ho, 2006). Thus, the interaction of attention and adaptation effects on performance can differ. Furthermore, attention can alter performance without altering appearance (Fuller \& Carrasco, 2006), so effects on performance and appearance do not necessarily covary.

Altered appearance is a well-known consequence of both adaptation and attention, yet no study has investigated the combined effects of adaptation and attention on appearance. Here, we test how adaptation and attention effects on appearance interact. We use speed perception as a 'model system', because we have a good understanding of its neural basis and of how adaptation and attention in isolation affect it.

\section{Additive vs. non-additive combinations of attention and adaptation}

We combined attention with adaptation and measured perceived speed using the method of constant stimuli (Figure 1). Observers judged the speed of two moving Gabor patterns-Test and Standard-while covert spatial attention was drawn to one of the stimuli by an uninformative, exogenous cue (Fuller et al., 2009; Turatto et al., 2007). An adaptor moving at one of two speeds, slow or fast, preceded either the cued or the uncued stimulus. Observers reported which stimulus they perceived as moving either faster (Experiment 1) or slower (Experiment 2).

Figure 2 shows the predicted outcomes for additive and non-additive effects. Here we refer to a non-additive combination as an interaction. Because perceived speed is generally not increased after adaptation with a slower speed (Smith, 1985; Thompson, 1981), we used a slow adaptor to measure a baseline attention effect. This allowed us to measure the 
magnitude of the attention effect with and without the influence of adaptation under the same conditions. The slow adaptor speed, which would yield no adaptation effect, was determined in preliminary testing. In this case, we predict the point of subjective equality (PSE) in the neutral condition to be at the point of physical equality (PPE; equal Test and Standard speeds), independent of whether the Standard or the Test location was adapted (Figure 2A, black curves). If attention increased perceived speed, cueing the Test would lead observers to select it as faster more often, resulting in a leftward shift of the PSE (blue curve): a physically slower Test speed would be perceived as being equal to the Standard speed. Conversely, cueing the Standard stimulus, the Test should be selected as faster less often, resulting in a rightward shift of the PSE: a physically faster Test speed would be perceived as being equal to the Standard speed (red curve).

The fast adaptor is expected to decrease perceived speed (Smith, 1985; Thompson, 1981) and should thus shift the neutral PSE to the left when the Standard is adapted and to the right if the Test is adapted (Figure 2B and C, black curve). If adaptation and attention combined additively, attention would shift the PSE by the same amount as in the slow adaptor condition, but relative to the shifted neutral (Figure 2B). Alternatively, if adaptation fatigued neuronal populations that mediate the attention effect, attention should not be able to exert its effect. In this case, adapting the Standard location (top panel) or the Test location (bottom panel) would reduce or cancel the effect of attention in the Standard cued condition or the Test cued condition, respectively (Figure 2C).

\section{Method \\ Observers}

Eight observers ( 4 female; mean age $=24.6$ years, standard deviation $=3.9$ ) participated in Experiment 1 and seven of them in Experiment 2. Because the aim of these experiments was to investigate the interaction between adaptation and attention rather than to test the effect of attention per se, we selected observers who had a significant effect of attention on perceived speed in a pre-experiment, in which we replicated the attention modulation of perceived speed (Fuller et al., 2009; Turatto et al., 2007). Four observers, inexperienced in visual psychophysical tasks, were paid for their participation; the other four were lab members. All observers, but one author, were naïve to the purpose of the study; all had normal or corrected-to-normal vision. The NYU Institutional Review Board approved all procedures. Two observers in Experiment 1 and one observer in Experiment 2 were excluded from all analysis due to large gaze deviations from fixation $\left(>1.5^{\circ}\right.$, see Eye Positions in Supplementary Materials).

\section{Apparatus}

Experiments were performed in a dark experimental room. Stimuli were presented on a calibrated, linearized CRT monitor (IBM P260, viewable area $40 \times 30 \mathrm{~cm}$, resolution $1 \mathrm{~cm} /{ }^{\circ}$ (32 pixels $/^{\circ}$ ), refresh rate $\left.85 \mathrm{~Hz}\right)$. Stimuli were presented on a gray background $\left(\sim 56 \mathrm{~cd} / \mathrm{m}^{2}\right)$. Observers used a chinrest positioned $57 \mathrm{~cm}$ from the monitor. Experiments were run on an Apple Macintosh computer (iMac) using a custom MATLAB (The MathWorks, Natick, MA, USA) script and the Psychophysics Toolbox extension (Brainard, 1997; Pelli, 1997). Eye positions were recorded using an infrared eye tracker (EyeLink CL, SR Research, Kanata, Ontario, Canada; see Eye Positions in Supplementary Materials).

\section{Stimuli}

Each trial started with the presentation of a dark $\left(<1 \mathrm{~cd} / \mathrm{m}^{2}\right)$ fixation square of $0.2^{\circ}$ side length at the center of the screen (Figure 1). After $300 \mathrm{~ms}$, an adaptor and a control stimulus were presented at $5.5^{\circ}$ left and right of and $2^{\circ}$ below fixation. The adaptor location (left or 
right) was constant within each block but alternated across blocks. The adaptor was a 1-cpd Gabor (Gaussian envelope of $1.24^{\circ}$ diameter at half height; Michelson contrast 60\%), moving upward behind the Gaussian aperture at either 2.82 or $6.56 \%$. The control stimulus was the same but moved rightward, i.e. orthogonal to the motion direction of Standard and Test, to keep stimulation constant at both locations without influencing perceived speed of the subsequent Test or Standard stimulus. In the first trial of each block, adaptor and control were presented for $50 \mathrm{~s}$, in each subsequent trial they were presented for top-up adaptation for $1.5 \mathrm{~s}$.

After an interstimulus interval (ISI) of $100 \mathrm{~ms}$, a $70 \mathrm{~ms}$ stationary cue was flashed. The $0.3 \times 0.7^{\circ}$ cue was a bright ( $100 \%$ contrast) rectangle; its position was either $2.5^{\circ}$ above the center of the Test position (Test cued condition), the Standard position (Standard cued condition), or at the fixation point (neutral condition). After an ISI of $40 \mathrm{~ms}$, Test and Standard stimuli were presented simultaneously at the same locations as the adaptor and control stimuli for $200 \mathrm{~ms}$. They had the same stimulus characteristics except for their speed. The Standard stimulus' speed was $4.3^{\circ} / \mathrm{s}$. The Test's speed was randomly chosen from 7-13 levels, adjusted for each observer separately for each adaptation condition. The medium level corresponded to the PSE and five levels were in the dynamic range of the psychometric function, defined as between the 10\%- and 90\%-threshold, in the corresponding adaptation condition in the training session. In the training session, the cue was always presented at fixation (neutral cue); the Test speed on each trial was randomly chosen from 13 levels equally spaced around the Standard speed (logarithmic scale, 1.21-15.24\%).

The locations of the cue, Test, and Standard stimulus were randomly and independently assigned on each trial. Thus, in a given trial, either the Test or the Standard stimulus could appear at the location where the adaptor had been presented (Test adapted and Standard adapted conditions, respectively), and could either be cued or not cued, so that all combinations of attention and adaptation conditions were counterbalanced.

\section{Procedure}

Each observer completed 680 trials in a 1-hr training session and 2720 or 3400 trials of the main experiment in 4 or 51 -hr sessions. At the beginning of each block, observers received instructions on the screen, either 'Please judge the stimuli in the second interval: Which stimulus moves faster?' (Experiment 1) or 'Please judge the stimuli in the second interval: Which stimulus moves slower?' (Experiment 2). Observers pressed the left-arrow or rightarrow key to indicate the left or the right stimulus, respectively (2-Alternative ForcedChoice (2-AFC) paradigm). Observers were instructed to respond as accurately and fast as possible, but response time was unlimited. Observers were instructed to keep fixation throughout each trial and the position of the right eye was recorded and analyzed offline (see Eye Positions in Supplementary Materials).

\section{Results}

\section{Effects of adaptation and attention on perceived speed are additive}

Experiment 1-Figures 3A-B show the results of a representative observer. For each combination of adaptation speed, adaptation condition (Standard or Test adapted), and cue condition, the proportion of trials in which the Test stimulus was selected as faster is plotted as a function of actual Test speed. These data were fit with a logistic function to derive PSE and slope estimates using the psignifit toolbox (http://bootstrap-software.org/psignifit/), which implements the maximum-likelihood method described by Wichmann and Hill, (2001). Mean $\mathrm{R}^{2}$ was 0.96 ( \pm 0.03 standard deviation) across all conditions ( 2 adaptor speeds x 2 adaptation conditions $x 3$ cue conditions) and 6 observers $(n=72)$. For the slow adaptor 
(left plots), the PSE in the neutral condition (black curve) did not differ from the PPE, either when the Standard stimulus (top plot) or the Test stimulus (bottom plot) was adapted. For the fast adaptor (right plots), the PSE in the neutral condition was shifted away from the actual Standard speed-to the left (top plot) or to the right (bottom plot), when the Standard or the Test stimulus was adapted, respectively-consistent with a decrease in perceived speed by adaptation. For both adaptation speeds, cueing the Test stimulus location (blue curve) shifted the PSE to the left of the neutral PSE, whereas cueing the Standard stimulus location (red curve) shifted the PSE to the right, consistent with an increase in perceived speed by attention. These effects were consistent across observers (Figures 3C, adaptation; 3D, attention).

A 3-way repeated measures ANOVA (2 adaptor speeds x 2 adaptation conditions $\mathrm{x} 3$ cue conditions) on the PSE revealed main effects of adaptation speed $(F(1,5)=9.08, p<0.05$; $\left.\eta^{2}=0.65\right)$, adaptation condition $\left(F(1,5)=65.87, p<0.0001 ; \eta^{2}=0.93\right)$, and cue condition $\left(F(1.06,5.30)=9.74, p<0.05 ; \eta^{2}=0.66\right)^{1}$. Neither the 3 -way interaction $(F(1.08,5.38)=1.61$, $\left.p>0.1 ; \eta^{2}=0.24\right)$ nor the interaction between adaptation speed and cue $(F(1.04,5.22)=1.57$, $\left.p>0.1 ; \eta^{2}=0.24\right)$ was significant. There was a significant interaction between adaptation speed and adaptation condition $\left(F(1,5)=30.11, p<0.005 ; \eta^{2}=0.85\right)$; an adaptation effect was only evident for the fast adaptor (Figure 3C). There was a marginal interaction between adaptation condition and cue $\left(F(1.10,5.49)=4.19, p<0.1 ; \eta^{2}=0.46\right)$; the cue effect was more pronounced for the Standard adapted condition than the Test adapted condition (Figure 3D).

Given that a non-additive interaction hypothesis would make different predictions for trials in which the Test location or the Standard location had been adapted (Figure 2C), we explored if an interaction between adaptation speed and cue condition would emerge in any of these two cases. The interaction between adaptation speed and cue condition was neither significant for the Standard nor for the Test adapted trials $\left(F(1.06,5.28)=1.58 ; \eta^{2}=0.24\right.$ and $F(1.05,5.27)=1.90 ; \eta^{2}=0.28$; both $\left.p>0.1\right)$.

Experiment 2-In principle, the effect of the cue in Experiment 1 could be explained by a bias of observers to select the cued stimulus. Such a response strategy would lead to the same shift of the psychometric function without implying a change in perceived speed. To control for response biases, we performed an experiment in which observers reported the slower instead of the faster stimulus. If observers were biased to select the cued stimulus, we would expect them to select the cued stimulus more often, whether asked for the faster or for the slower stimulus. Such a bias would cause the PSE to shift in opposite directions for these two different instructions. In contrast, an attention increase in perceived speed would shift the PSE in the same direction independent of instruction. Thus, comparing the cue effect in these two tasks can be used to estimate the attention effect without any response bias contribution (Anton-Erxleben et al., 2007; Carrasco et al., 2004; Fuller \& Carrasco, 2006; Gobell \& Carrasco, 2005; Ling \& Carrasco, 2007; Liu et al., 2009; Montagna \& Carrasco, 2006; Turatto et al., 2007).

Figures 4A-B show the results of a representative observer. Data were analyzed as in Experiment 1. Mean $\mathrm{R}^{2}$ was 0.95 ( \pm 0.06 standard deviation) across all 12 conditions and 6 observers $(\mathrm{n}=72)$. As in Experiment 1, the fast adaptor decreased perceived speed, but there was no evidence for an adaptation effect with the slow adaptor (Figure 4A-C). For each adaptation condition, the cue shifted the PSE consistent with an increase in perceived speed by attention (Figure 4A-B, D).

${ }^{1}$ Whenever Mauchly's test of sphericity indicated a violation of the assumption of sphericity, we report Greenhouse-Geisser-corrected p-values. 
A 3-way repeated measures ANOVA (2 adaptor speeds x 2 adaptation conditions $\mathrm{x} 3$ cue conditions) on the PSE revealed main effects of adaptation condition $(F(1,5)=23.89$; $\left.p=0.005 ; \eta^{2}=0.83\right)$ and cue $\left(F(1.06,5.32)=29.84 ; p<0.005 ; \eta^{2}=0.86\right)$, but no main effect of adaptation speed $\left(F(1,5)=3.98 ; p>0.1 ; \eta^{2}=0.44\right)$. Neither the 3 -way interaction $(F(1.12,5.61)$; $\left.p>0.1 ; \eta^{2}=0.23\right)$ nor the interaction between adaptation speed and cue $(F(1.11,5.56) ; p>0.1$; $\eta^{2}=0.35$ ) was significant. There was a significant interaction of adaptation speed and adaptation condition $\left(F(1,5)=14.67 ; p<0.05 ; \eta^{2}=0.75\right)$; an adaptation effect was only evident for the fast adaptor (Figure 4C). There was also a significant interaction of adaptation condition and cue $\left(F(2,10)=4.23 ; p<0.05 ; \eta^{2}=0.46\right)$; the cue effect was more pronounced for the Standard adapted condition than the Test adapted condition (Figure 4D).

As in Experiment 1, we analyzed the interaction of adaptation speed and the cue separately for Standard adapted and Test adapted trials. Again, the interaction between adaptation speed and cue condition was neither significant for Standard nor for Test adapted trials $\left(F(1.06,5.29)=2.27 ; \eta^{2}=0.31\right.$ and $F(2,10)=0.79 ; \eta^{2}=0.14 ;$ both $\left.p>0.1\right)$.

In order to directly compare the magnitude of the attention effect between both experiments, we calculated the average shift between the Standard cued and Test cued PSE over all adaptation conditions for those observers whose data were included in both experiments. This average attention effect was not significantly different between the two experiments (two-tailed paired t-test; $t(4)=0.57 ; p>0.1$; Cohen's $d=0.32$ ), demonstrating that the cue effect cannot be explained by a response bias. For the effects of adaptation and attention on the slopes of the psychometric functions see Slope Analysis in Supplementary Materials.

\section{Discussion}

In summary, two experiments show that adaptation does not modulate the effect of attention on perceived speed, indicating, for the first time, that adaptation and attention on appearance combine additively.

In both experiments, the fast adaptor decreased perceived speed whereas the slow adaptor had no effect on perceived speed. These findings are in line with previous studies: Although generally, adaptation to a particular stimulus feature shifts perception of similar stimulus features away from the adapted feature (Blakemore et al., 1970; Levinson \& Sekuler, 1976), for speed the effects can be asymmetric so that speeds below the adaptor speed are further reduced whereas higher speeds are not further increased (Smith, 1985; Thompson, 1981). Attention increased perceived speed, also consistent with previous studies (Fuller et al., 2009; Turatto et al., 2007). Most importantly, the magnitude of this attention effect did not vary with adaptor speed. The additive combination of adaptation and attention could indicate that distinct neuronal populations or distinct mechanisms within the same population underlie each effect.

\section{Which mechanisms mediate adaptation and attention effects on perceived speed?}

The medial temporal area (MT) is a likely candidate for effects of adaptation and attention: Speed selective cells are common in MT (Lingnau, Ashida, Wall, \& Smith, 2009; J. Liu \& Newsome, 2003; Priebe, Cassanello, \& Lisberger, 2003), and the perception of speed is related to the firing of MT neurons (Liu \& Newsome, 2005). Several computational models explain how perception of stimulus speed can be derived from population activity (for a review, see Burr \& Thompson, 2011).

Changes in perception after adaptation have been associated with shifts in neuronal selectivity (Jin et al., 2005; Krekelberg et al., 2006; Levinson \& Sekuler, 1976). Covert spatial attention is generally associated with enhanced neuronal activity (Beck \& Kastner, 
2009; Martinez-Trujillo \& Treue, 2002; Reynolds, Pasternak, \& Desimone, 2000; Williford $\&$ Maunsell, 2006). In a labeled-line framework, modulation of perceived speed is likely to involve a shift instead of a mere increase in neuronal population responses: Specifically, an increase of apparent speed might be correlated to an enhanced activity of neuronal populations, which are tuned to a higher speed, and/or a relative decrease of activity of neurons preferring a lower speed. Similarly, attention has been suggested to modulate perceived spatial frequency (Abrams et al., 2010; Gobell \& Carrasco, 2005) or perceived flicker rate (Montagna \& Carrasco, 2006) by selectively changing the sensitivity of particular spatial-frequency channels or temporal frequency channels, respectively, and thereby reweighting the population response.

The Bayesian model assumes that perceived speed is the product of a likelihood distribution, centered at the true stimulus speed, and a prior distribution, centered at zero speed, which biases perception towards slower speeds depending on signal strength (Weiss, Simoncelli, \& Adelson, 2002). Neuronal populations can represent likelihoods by noisy spiking activity (Ma, Beck, Latham, \& Pouget, 2006), and priors by the distribution of neurons' selectivity and tuning width according to the frequency with which certain stimulus properties are encountered in the environment (Ganguli \& Simoncelli, 2010). A Bayesian decoder can combine prior and likelihood to get an estimate of the stimulus by computing a weighted average of preferred stimuli (Ganguli \& Simoncelli, 2012), similar to vector averaging mechanisms often employed in labeled-line models. The Bayesian model can explain the increase in perceived speed at higher contrasts, and could explain the increase of perceived speed with attention: Given that attention has been shown to enhance the stimulus representation (Carrasco, 2011; Reynolds \& Chelazzi, 2004; Treue, 2003), from a Bayesian viewpoint, attention might enhance the signal-to-noise ratio of neurons tuned to the stimulus' speed by increasing their gain, so that perception is shifted away from a zeromotion prior.

Thus, the labeled-line and the Bayesian frameworks that have been developed to account for speed perception in other contexts, could also explain the effect of attention on perceived speed.

\section{Interaction of adaptation and attention}

Previous studies have investigated how attention affects the adaptation process (Alais \& Blake, 1999; Hudson et al., 2009; Lankheet \& Verstraten, 1995; Ling \& Carrasco, 2006; Liu et al., 2007). However, only a couple of studies have investigated how adaptation affects subsequent effects of attention on performance, and have revealed additive (Pestilli et al., 2007) and non-additive effects (Barbot, Montagna, \& Carrasco, 2011; Carrasco, Loula et al., 2006) in different visual domains.

The present study is the first to investigate the interaction of adaptation and attention effects on appearance. By manipulating attention during different adaptation states, we find that adaptation and attention affect appearance independently. Thus, consistent with the contrast sensitivity study (Pestilli et al., 2007), we provide converging behavioral evidence that adaptation is not merely a neuronal fatigue effect: Were neuronal responses in the adapted state saturated due to fatigue, attention should not be able to restore contrast sensitivity or perceived speed. Rather, it seems that attention can 'reset' the system when adaptation is present: Here, adapting the Standard stimulus' location shifts perception away from the physical Standard speed, whereas allocating attention to the Standard stimulus shifts perception back towards the physical Standard speed (Figures 3A \& 4A). When the Test stimulus' location is adapted, attention on the Test stimulus shifts the PSE back to the Test speed that is physically equal to the Standard speed (Figures 3B \& 4B). Both of these effects are consistent with attention restoring the base level speed perception after adaptation. Note, 
however, that this does not imply that attention leads to veridical perception: In the case without adaptation, attention leads to speed overestimation.

In contrast, the effects of adaptation on spatial resolution are more consistent with a fatigue explanation: In a texture segmentation task, performance is determined by the proportional activation of low- and high-spatial frequency filters. After adaptation to a high-spatial frequency pattern, both performance and the attention effects on performance are modulated consistent with a decreased availability of high-spatial frequency filters, suggesting that attention enhances spatial resolution by shifting population sensitivity towards higher spatial frequencies (Carrasco, Loula et al., 2006).

\section{Conclusion}

In summary, we show for the first time that adaptation does not alter the effect of attention on perceived speed. Given that for spatial frequency, adaptation and attention effects on performance are not independent, it would be interesting in future studies to investigate how adaptation state modulates the attention effect on spatial frequency appearance. In line with previous research, our findings support the view that adaptation is not simply a neuronal fatigue effect. Instead, attention can 'reset' the system in a dynamic fashion. Both adaptation and attention lead to relatively short-term plastic changes by which the visual system adjusts to current environmental demands either related to sensory history or to behavioral relevance, thereby prioritizing important over unimportant information. The finding that the effect of attention on perceived speed is not modulated by the adaptation state reveals independent effects of adaptation and attention, two adaptive mechanisms that help us allocate limited resources.

\section{Supplementary Material}

Refer to Web version on PubMed Central for supplementary material.

\section{Acknowledgments}

We thank Franco Pestilli and the current members of the Carrasco lab for helpful comments. This research was supported by a Feodor-Lynen Research Fellowship, Alexander-von-Humboldt Foundation, and NRSA 1F32EY021420 to KAE, NIH R01-EY019693 and NIH EY016200 to MC.

\section{References}

Abrams J, Barbot A, Carrasco M. Voluntary attention increases perceived spatial frequency. Atten Percept Psychophys. 2010; 72:1510-1521. [PubMed: 20675797]

Alais D, Blake R. Neural strength of visual attention gauged by motion adaptation. Nat Neurosci. 1999; 2(11):1015-1018. [PubMed: 10526342]

Anton-Erxleben K, Abrams J, Carrasco M. Evaluating comparative and equality judgments in contrast perception: Attention alters appearance. J Vis. 2010; 10(11):6, 1-22. [PubMed: 20884501]

Anton-Erxleben K, Henrich C, Treue S. Attention changes perceived size of moving visual patterns. J Vis. 2007; 7(11):5, 1-9. [PubMed: 17997660]

Barbot A, Montagna B, Carrasco M. Endogenous attention optimizes spatial resolution depending on task demands. Perception. 2011; 40(ECVP Abstract Supplement):191. [PubMed: 21650092]

Beck DM, Kastner S. Top-down and bottom-up mechanisms in biasing competition in the human brain. Vision Res. 2009; 49(10):1154-1165. [PubMed: 18694779]

Blakemore C, Nachmias J, Sutton P. The perceived spatial frequency shift: evidence for frequencyselective neurones in the human brain. J Physiol. 1970; 210(3):727-750. [PubMed: 5499822]

Brainard DH. The Psychophysics Toolbox. Spat Vis. 1997; 10:433-436. [PubMed: 9176952] 
Burr D, Thompson P. Motion psychophysics: 1985-2010. Vision Res. 2011; 51(13):1431-1456. [PubMed: 21324335]

Carrasco M. Visual attention: The past 25 years. Vision Res. 2011; 51:1484-1525. [PubMed: 21549742]

Carrasco M, Giordano AM, McElree B. Attention speeds processing across eccentricity: feature and conjunction searches. Vision Res. 2006; 46(13):2028-2040. [PubMed: 16481020]

Carrasco M, Ling S, Read S. Attention alters appearance. Nat Neurosci. 2004; 7(3):308-313. [PubMed: 14966522]

Carrasco M, Loula F, Ho YX. How attention enhances spatial resolution: evidence from selective adaptation to spatial frequency. Percept Psychophys. 2006; 68(6):1004-1012. [PubMed: 17153194]

Carrasco M, Penpeci-Talgar C, Eckstein M. Spatial covert attention increases contrast sensitivity across the CSF: support for signal enhancement. Vision Res. 2000; 40(10-12):1203-1215. [PubMed: 10788636]

Fuller S, Carrasco M. Exogenous attention and color perception: performance and appearance of saturation and hue. Vision Res. 2006; 46(23):4032-4047. [PubMed: 16979690]

Fuller S, Park Y, Carrasco M. Cue contrast modulates the effects of exogenous attention on appearance. Vision Res. 2009; 49(14):1825-1837. [PubMed: 19393260]

Ganguli D, Simoncelli EP. Implicit encoding of prior probabilities in optimal neural populations. Adv Neural Information Processing Systems. 2010; 23:658-666.

Ganguli, D.; Simoncelli, EP. Neural implementation of Bayesian inference using efficient population codes. Paper presented at the Computational and Systems Neuroscience; 2012.

Giordano AM, McElree B, Carrasco M. On the automaticity and flexibility of covert attention: a speed-accuracy trade-off analysis. J Vis. 2009; 9(3):30, 31-10. [PubMed: 19757969]

Gobell J, Carrasco M. Attention alters the appearance of spatial frequency and gap size. Psychol Sci. 2005; 16(8):644-651. [PubMed: 16102068]

Herrmann K, Montaser-Kouhsari L, Carrasco M, Heeger DJ. When size matters: attention affects performance by contrast or response gain. Nat Neurosci. 2010; 13(12):1554-1559. [PubMed: 21057509]

Hudson AE, Schiff ND, Victor JD, Purpura KP. Attentional modulation of adaptation in V4. Eur J Neurosci. 2009; 30(1):151-171. [PubMed: 19558603]

Jin DZ, Dragoi V, Sur M, Seung HS. Tilt aftereffect and adaptation-induced changes in orientation tuning in visual cortex. J Neurophysiol. 2005; 94(6):4038-4050. [PubMed: 16135549]

Kohn A. Visual adaptation: physiology, mechanisms, and functional benefits. J Neurophysiol. 2007; 97(5):3155-3164. [PubMed: 17344377]

Krekelberg B, van Wezel RJ, Albright TD. Adaptation in macaque MT reduces perceived speed and improves speed discrimination. J Neurophysiol. 2006; 95(1):255-270. [PubMed: 16192331]

Lankheet MJ, Verstraten FA. Attentional modulation of adaptation to two-component transparent motion. Vision Res. 1995; 35(10):1401-1412. [PubMed: 7645269]

Lennie P. The cost of cortical computation. Curr Biol. 2003; 13(6):493-497. [PubMed: 12646132]

Levinson E, Sekuler R. Adaptation alters perceived direction of motion. Vision Res. 1976; 16(7):779781. [PubMed: 960603]

Ling S, Carrasco M. When sustained attention impairs perception. Nat Neurosci. 2006; 9(10):12431245. [PubMed: 16964254]

Ling S, Carrasco M. Transient covert attention does alter appearance: a reply to Schneider (2006). Percept Psychophys. 2007; 69(6):1051-1058. [PubMed: 18018987]

Lingnau A, Ashida H, Wall MB, Smith AT. Speed encoding in human visual cortex revealed by fMRI adaptation. J Vis. 2009; 9(13):3, 1-14. [PubMed: 20055536]

Liu J, Newsome WT. Functional organization of speed tuned neurons in visual area MT. J Neurophysiol. 2003; 89(1):246-256. [PubMed: 12522176]

Liu J, Newsome WT. Correlation between speed perception and neural activity in the middle temporal visual area. J Neurosci. 2005; 25(3):711-722. [PubMed: 15659609] 
Liu T, Abrams J, Carrasco M. Voluntary attention enhances contrast appearance. Psychol Sci. 2009; 20(3):354-362. [PubMed: 19254239]

Liu T, Fuller S, Carrasco M. Attention alters the appearance of motion coherence. Psychon Bull Rev. 2006; 13(6):1091-1096. [PubMed: 17484441]

Liu T, Larsson J, Carrasco M. Feature-based attention modulates orientation-selective responses in human visual cortex. Neuron. 2007; 55(2):313-323. [PubMed: 17640531]

Lu ZL, Dosher BA. External noise distinguishes attention mechanisms. Vision Res. 1998; 38(9):11831198. [PubMed: 9666987]

Luck SJ, Hillyard SA, Mouloua M, Woldorff MG, Clark VP, Hawkins HL. Effects of spatial cuing on luminance detectability: psychophysical and electrophysiological evidence for early selection. J Exp Psychol Hum Percept Perform. 1994; 20(4):887-904. [PubMed: 8083642]

Ma WJ, Beck JM, Latham PE, Pouget A. Bayesian inference with probabilistic population codes. Nat Neurosci. 2006; 9(11):1432- 1438. [PubMed: 17057707]

Martinez-Trujillo J, Treue S. Attentional modulation strength in cortical area MT depends on stimulus contrast. Neuron. 2002; 35(2):365-370. [PubMed: 12160753]

Montagna B, Carrasco M. Transient covert attention and the perceived rate of flicker. J Vis. 2006; 6(9):955-965. [PubMed: 17083287]

Montagna B, Pestilli F, Carrasco M. Attention trades off spatial acuity. Vision Res. 2009; 49(7):735745. [PubMed: 19385088]

Movshon JA, Lennie P. Pattern-selective adaptation in visual cortical neurones. Nature. 1979; 278(5707):850-852. [PubMed: 440411]

Ohzawa I, Sclar G, Freeman RD. Contrast gain control in the cat visual cortex. Nature. 1982; 298(5871):266-268. [PubMed: 7088176]

Pelli DG. The VideoToolbox software for visual psychophysics: Transforming numbers into movies. Spat Vis. 1997; 10:437-442. [PubMed: 9176953]

Pestilli F, Viera G, Carrasco M. How do attention and adaptation affect contrast sensitivity? J Vis. 2007; 7(7):9, 1-12. [PubMed: 17685805]

Priebe NJ, Cassanello CR, Lisberger SG. The neural representation of speed in macaque area MT/V5. J Neurosci. 2003; 23(13):5650-5661. [PubMed: 12843268]

Reynolds JH, Chelazzi L. Attentional modulation of visual processing. Annu Rev Neurosci. 2004; 27:611-647. [PubMed: 15217345]

Reynolds JH, Pasternak T, Desimone R. Attention increases sensitivity of V4 neurons. Neuron. 2000; 26(3):703-714. [PubMed: 10896165]

Smith AT. Velocity coding: evidence from perceived velocity shifts. Vision Res. 1985; 25(12):19691976. [PubMed: 3832622]

Störmer VS, McDonald JJ, Hillyard SA. Cross-modal cueing of attention alters appearance and early cortical processing of visual stimuli. Proc Natl Acad Sci USA. 2009; 106:22456-22461. [PubMed: 20007778]

Thompson P. Velocity after-effects: the effects of adaptation to moving stimuli on the perception of subsequently seen moving stimuli. Vision Res. 1981; 21(3):337-345. [PubMed: 7269311]

Treue S. Climbing the cortical ladder from sensation to perception. Trends Cogn Sci. 2003; 7(11):469471. [PubMed: 14585436]

Turatto M, Vescovi M, Valsecchi M. Attention makes moving objects be perceived to move faster. Vision Res. 2007; 47(2):166-178. [PubMed: 17116314]

Webster MA. Adaptation and visual coding. J Vis. 2011; 11(5)

Weiss Y, Simoncelli EP, Adelson EH. Motion illusions as optimal percepts. Nat Neurosci. 2002; 5(6): 598-604. [PubMed: 12021763]

Wichmann FA, Hill NJ. The psychometric function: I. Fitting, sampling, and goodness of fit. Percept Psychophys. 2001; 63(8):1293-1313. [PubMed: 11800458]

Williford T, Maunsell JH. Effects of spatial attention on contrast response functions in macaque area V4. J Neurophysiol. 2006; 96(1):40-54. [PubMed: 16772516]

Yeshurun Y, Carrasco M. Attention improves or impairs visual performance by enhancing spatial resolution. Nature. 1998; 396(6706):72-75. [PubMed: 9817201] 


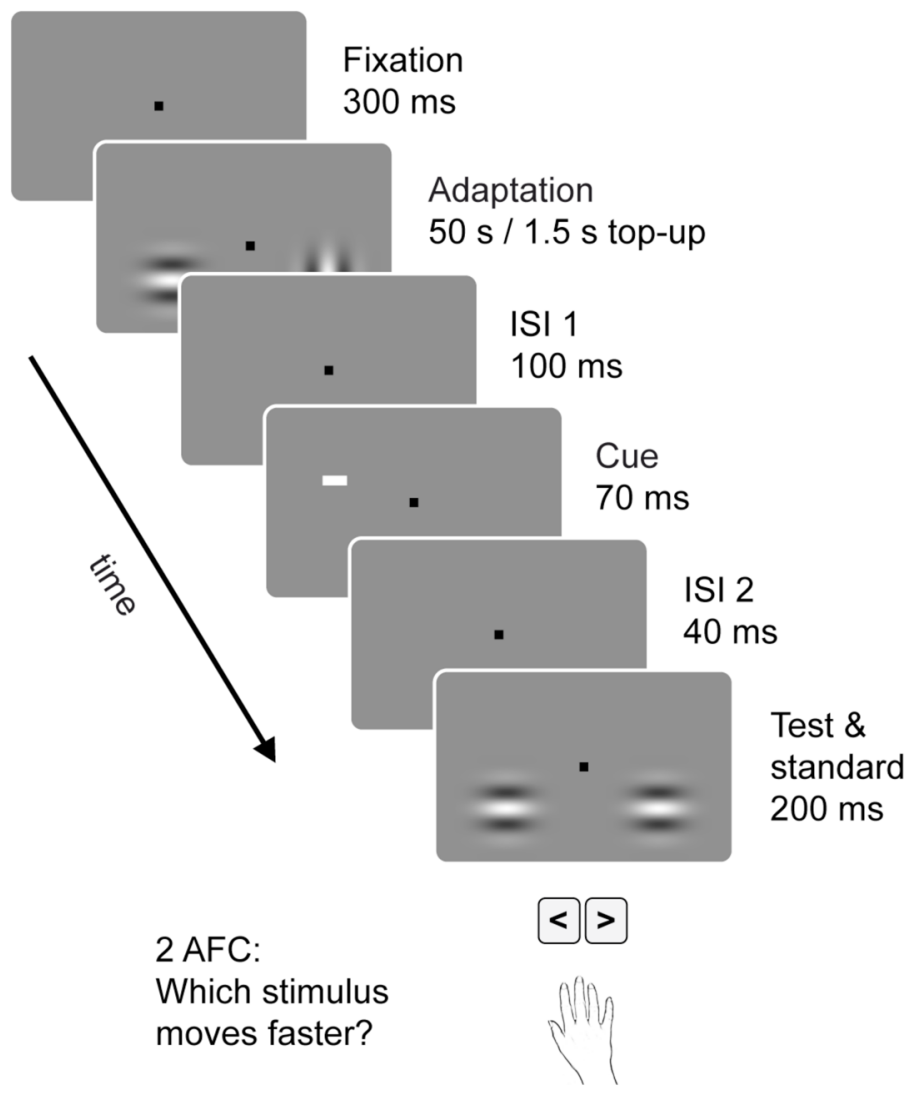

Figure 1.

Experimental design. After a fixation period, two adaptors were presented at the subsequent Test and Standard's location, one moving in the same direction as Test and Standard, the other moving orthogonally. After an interstimulus interval (ISI), a cue was flashed either at fixation (neutral condition) or $2.5^{\circ}$ above the stimuli's location (Test cued and Standard cued conditions). After another ISI, two moving Gabors (Test and Standard) were presented. Standard speed was kept constant while Test speed varied across trials in a 2-alternative forced choice task (2AFC). Observers reported either which stimulus was moving faster (Experiment 1) or slower (Experiment 2). Drawings are not to scale. 

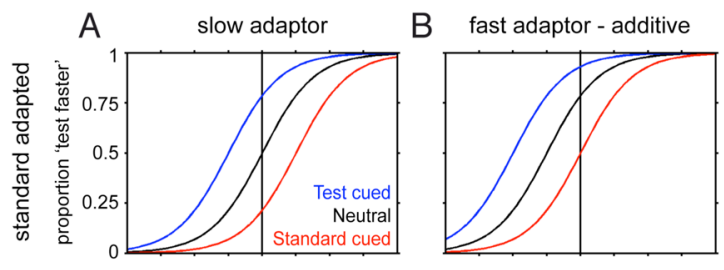

C fast adaptor - non-additive
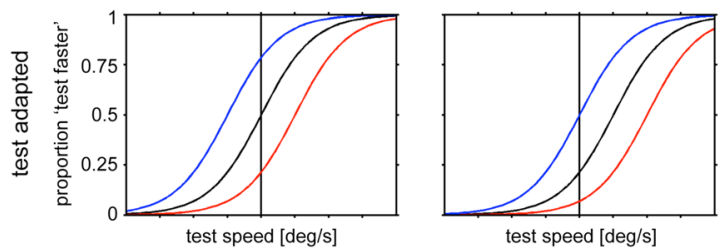

test speed [deg/s]
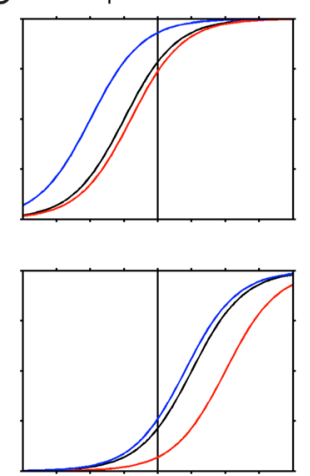

test speed [deg/s]

Figure 2.

Hypothetical results for additive and non-additive combinations of attention and adaptation. A: Adaptation with slow speed. Proportion of reporting the Test as being faster for Standard adapted (upper row) and Test adapted (bottom row) trials. Blue: Test cued, black: neutral, red: Standard cued condition. Vertical solid line marks the physical Standard speed. B:

Adaptation with fast speed assuming additive effects. Same format as A. C: Adaptation with fast speed assuming non-additive effects. Same format as A. 


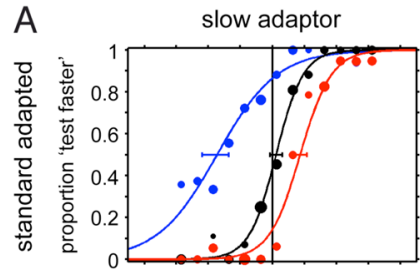

B

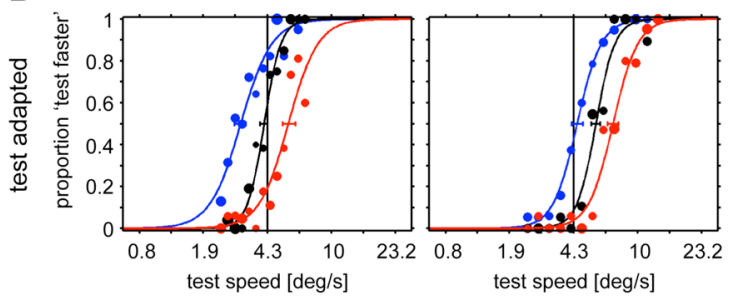

fast adaptor

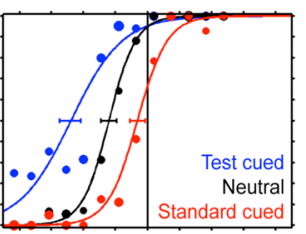

\section{D}

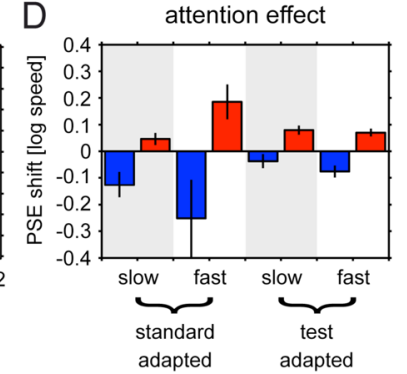

\section{Figure 3.}

Results Experiment 1. A: Proportion of reporting the Test as being faster for a single observer in the Standard adapted condition after adaptation with the slow (left) and fast (right) adaptor speed. Blue: Test cued, black: neutral, red: Standard cued condition. Marker size reflects the number of trials for each data point. Solid lines are fits with a logistic function. Error bars are $95 \%$ confidence intervals derived from bootstrapping. Vertical solid line marks the physical Standard speed. B: Data from the same observer in the Test adapted condition. Same format as A. C: Average adaptation effect across observers ( $n=6)$. Shift of the PSE in the neutral condition relative to the physical Standard speed. Error bars are standard error of the mean (SEM). Same color code as A and B. D: Average attention effect across observers $(n=6)$. Shift of the PSE relative to the neutral condition for the slow and fast adaptor in the Standard adapted and Test adapted condition. Same format as C. 

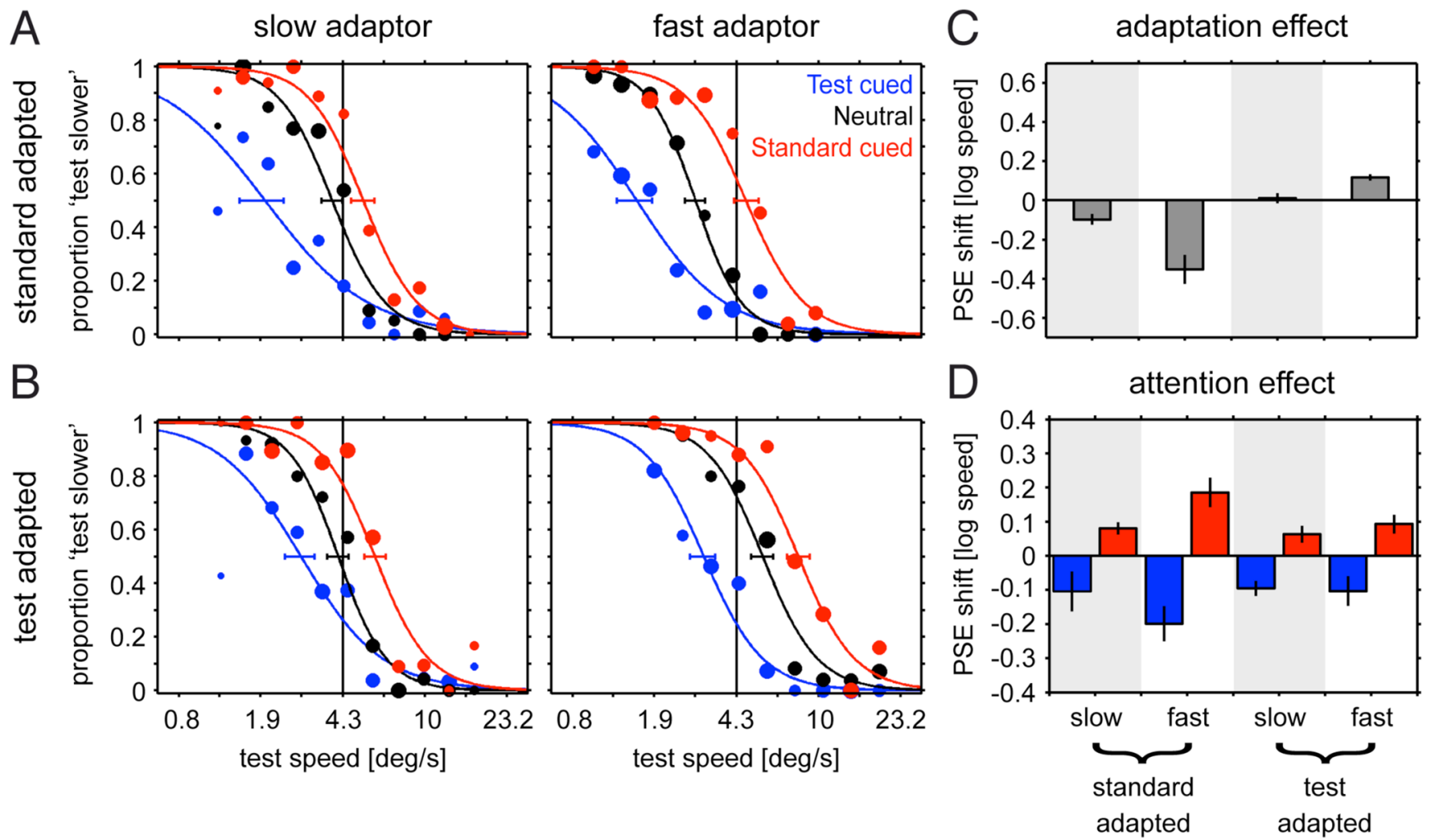

Figure 4.

Results Experiment 2. A: Proportion of reporting the Test as being slower for a single observer in the Standard adapted condition after adaptation with the slow (left) and fast (right) adaptor speed. Blue: Test cued, black: neutral, red: Standard cued condition. Marker size reflects the number of trials for each data point. Solid lines are fits with a logistic function. Error bars are 95\% confidence intervals derived from bootstrapping. Vertical solid line marks the physical Standard speed. B: Data from the same observer in the Test adapted condition. Same format as A. C: Average adaptation effect across observers $(n=6)$. Shift of the PSE in the neutral condition relative to the physical Standard speed. Error bars are standard error of the mean (SEM). Same color code as A and B. D: Average attention effect across observers $(n=6)$.. Shift of the PSE relative to the neutral condition for the slow and fast adaptor in the Standard adapted and Test adapted condition. Same format as C. 